\title{
The Effect of myo-Inositol on Pyruvate Decarboxylase ${ }^{1}$
}

\author{
Takako Tomita, Isao Tomita \\ and Tatsuo OzaWA ${ }^{2}$ \\ Shizuoka College of Pharmaceutical Sciences \\ 160 Oshika, Shizuoka (Post No. 420)
}

(Received January 10, 1969)

\begin{abstract}
Saccharomyces carlsbergensis 4228 (ATCC 9080) was cultivated for 24 hours in four different media containing $0,50,150$ and $500 \mu \mathrm{g}$ of myo-inositol per $20 \mathrm{ml}$. The growth of the yeast and the specific activity of pyruvate decarboxylase extracted from the respective cell preparations were found to be proportional to the amount of inositol in the medium. The level of acetoin in the culture medium decreased reversely with the concentration of inositol. Both acetoin accumulation and pyruvate decarboxylase activity of the yeast were influenced significantly by the lots of Casamino acid used as a nitrogen source in the basal medium. Among five Casamino acid peparations tested, R 69 and NR 1219 gave the highest and the lowest "acetoin ratio" respectively. With the amino acid mixtures reconstituted on the basis of amino acid analysis of the two Casamino acids, the patterns of acetoin accumulation were the same as those obtained with respective Casamino acid. The increasing responses of pyruvate decarboxylase to the concentration of inositol in the medium observed with Casamino acid, however, were not ireproduced with the reconstituted amino acid mixtures. Variation of the acetoin level in the culture medium seems to be caused by the composition of amino acids in the lots and the activation of pyruvate decarboxylase by inositol seems to be ascribed to an unknown substance in Casamino acid.
\end{abstract}

It has been reported that the activity of pyruvate decarboxylase (PDC) (EC 4.1.1.1) in a thiamine-deficient mutant of Saccharomyces cerevisiae was increased upon the addition of a component of the coenzyme, thiamine, to the medium (1). In Saccharomyces carlsbergensis 4228 (ATCC 9080) which requires myo-inositol for the normal growth, the specific activity of PDC increased proportionally to the increased amounts of inositol added to the medium (2). The effect of inositol on PDC was studied in detail and the enhancement of the enzyme activity was

\footnotetext{
1 The Role of myo-Inositol in Metabolic Control. VI.

2 富田多嘉子, 富田 勲, 小沢樹夫.
} 
found to be strongly influenced by the source of Casamino acids used; small increase of PDC activity was found with a certain Casamino acid and no significant increase was observed when pure amino acid mixture was used as a nitrogen source.

The present communication describes the evidence that the rate of PDC synthesis or activation depends upon an unknown factor in Casamino acid as well as the amount of inositol in the medium.

\section{Methods ANd Materials}

S. carlsbergensis strain 4228 (ATCC 9080) statically cultivated at $30^{\circ}$ for 20 hours in the complete medium (3) was used to inoculate the media containing 0 , 50,150 and $500 \mu \mathrm{g}$ of inositol per $20 \mathrm{ml}$ (medium 1,2,3 and 4), respectively, in $50 \mathrm{ml}$ Erlenmyer flasks. They were incubated at $30^{\circ}$ with shaking. The cells used for the test of PDC were harvested at 24 hours and washed three times with water before use. PDC was extracted with $0.5 M$ phosphate buffer ( $\mathrm{pH} 7.2$ ) from the washed dried cells according to the method of Aoshima (4). After removal of the cells, the supernatant was centrifuged again at $8,000 \mathrm{rpm}$ for 15 minutes and the activity was measured on the crude extracts by $\mathrm{CO}_{2}$ evolution (5) or acetoin formation (6). Acetoin released in the culture media was determined by the method of Westerfeld (7).

Vitamin-free Casamino acids (acid-hydrolyzed) was purchased from Nissui Chemicals and their amino acid analysis was conducted with Shibata Amino Acid Analyzer (Model 600).

Amino acids were purchased from Kyowa Hakko Chemicals and inositol was given by Dr. G. Kimura, the Central Research Laboratory, Tokyo High Pressure Industry, Inc.

\section{RESULTS}

\section{The Relation between PDC Activity and the Concentration of Inositol in the Medium}

The broken line in Fig. 1 shows the growth response of the yeast grown on four different media with increasing concentrations of inositol $(0,50,150$ and $500 \mu \mathrm{g}$ per $20 \mathrm{ml}$ medium, designated medium $1,2,3$ and 4 respectively). The growth on the depleted medium (medium 1 ) at $30^{\circ}$ with shaking was repressed to about $1 / 4$ that in the complete medium (medium 4) in 24-hour cultivation and to $1 / 5$ in 48 hour cultivation.

The dashed line represents the amount of acetoin in the supernatants of four different 24-hour culture media. The level of acetoin in medium $1(0.885 \mu$ moles per mg of dry cell weight) was remarkably high compared with those in medium $2(0.088)$, medium $3(0.028)$ and medium $4(0.030)$. The ratios of acetoin accumulation in medium 1 to medium 4 are 30 in 24-hour cultivation and 250 in 48-hour cultivation in this experiment, where Casamino acid NR 1219 was used.

The shaded columns in Fig. 1. show the activity of PDC extracted from the 24-hour yeast cells grown in four different media. PDC activities of the enzyme extracts were $0.009 \mu$ mole $\mathrm{CO}_{2} / \mathrm{mg}$ protein per min (medium 1), 0.05 (medium 2), 


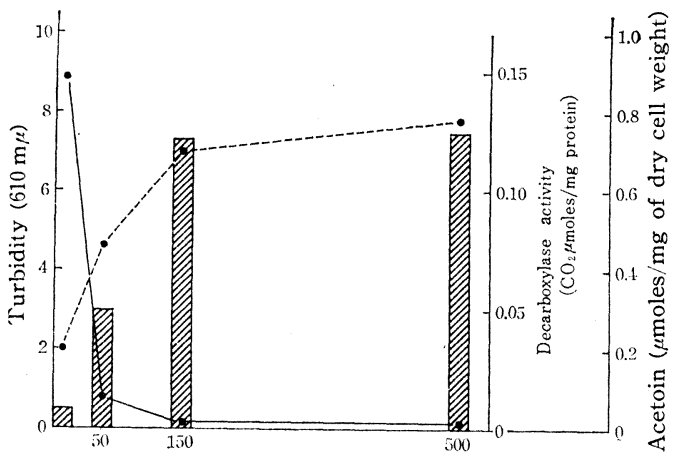

myo-Inositol supplementation ( $\mu \mathrm{g} / 20 \mathrm{ml}$ medium)

FIG. 1 Response Curves of Growth, Acetoin Accumulation and Pyruvate Decarboxylase Activity in Saccharomyces carlsbergensis to Inositol Supplementation to Medium (Casamino Acid NR 1219)

Saccharomyces carlsbergensis were cultivated in each medium for 24 hours at $30^{\circ}$ with shaking.

The broken line indicates turbidities at $610 \mathrm{~m} \mu$ and the dashed line the amounts of acetoin in the culture media. The shaded columns show the pyruvate decarboxylase activities extracted from 24-hour growth cells.
0.123 (medium 3) and 0.128 (medium 4). The activities measured by acetoin formation were $0,0.186$, 0.977 and $0.924 \mu$ moles acetoin $/ \mathrm{mg}$ protein per hour respectively.

It is evident from the figure that the changes of the activities are mainly controlled by the concentration of inositol in the medium. In contrast to large acetoin accumulation, the specific activity of PDC in the deficient cells was very low and addition of inositol to the reaction system for $\mathrm{PDC}$ activity test $(25 \mu \mathrm{g}$ inositol per $\mathrm{ml}$ reaction mixture) could not restore the ac tivity of the enzyme which was extracted from the yeast cells grown on medium 1 or 2 . As a threshold of inositol effect in the cells seems to be around $150 \mu \mathrm{g} / 20 \mathrm{ml}$ medium from the growth curve and acetoin accumulation. No difference in PDC activities between the cells grown

in medium 3 and 4 could be explained from this point of view.

2. The Effects of Casamino Acid on Growth, Acetoin Accumulation and PDC Activity of the Yeast

It was reported in the previous paper (3) that Casamino acids used as a nitrogen source in the medium could vary the acetoin accumulation ratio (the deficient to normal yeast) significantly according to the lots. Among five Casamino acids tested, two extremes, lot R 69 with the highest ratio and NR 1219 with the lowest ratio, were chosen to see the cause of difference in acetoin accumulation. The experiment shown in Fig. 1 was all carried out with Casamino acid NR 1219 as Hitrogen source in the medium. In Fig. 2 is given the result obtained by the repeated experiment using Casamino acid R 69 as nitrogen source.

The growth response on $\mathrm{R} 69$ to the increasing concentrations of inositol was quite identical to that on NR 1219 , but there were considerable differences in the patterns of acetoin accumulation and PDC activity between the two. The acetoin level was $2.80 \mu \mathrm{moles} / \mathrm{mg}$ of dry cell weight in medium 1 and 2.61 in medium 2 with R 69 which were 3.2 and 29.6 times those in the corresponding media with NR 1219 in 24-hour cultivation, while negligible amount of acetoin $(0.02-0.03 \mu$ mole) was detected in medium 3 and medium 4. Thus the acetoin ratios with $\mathrm{R} 69$ (100 at $24 \mathrm{hr}$ and 402 at $48 \mathrm{hr}$ ) were much greater than those with NR 1219.

PDC activity of the yeast grown on $\mathrm{R} 69$ was $0.127,0.085,0.174$ and 0.243 $\mu$ mole $\mathrm{CO}_{2} / \mathrm{mg}$ protein per min (or $0.480,0.264,1.206$ and $1.452 \mu$ moles of acetoin $/ \mathrm{mg}$. 


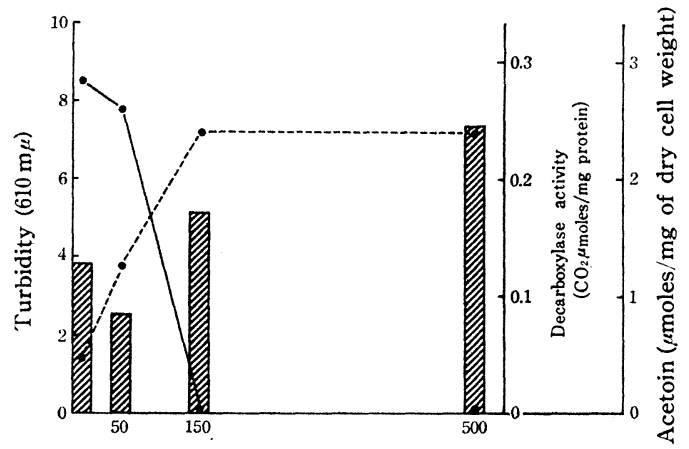

myo-Inositol supplementation ( $\mu \mathrm{g} / 20 \mathrm{ml}$ medium)

FIG. 2 Response Curves of Growth, Acetion Accumulation and Pyruvate Decarboxylase Activity in Saccharomyces carlsbergensis to Inositol Supplementation to Medium (Casamino Acid R 691

Saccharomyces carlsbergensis were cultivated in each medium for 24 hours at $30^{\circ}$ with shaking

The broken line indicates turbidities at $610 \mathrm{~m} \mu$ and the dashed line the amounts of acetoin in the culture media. The shaded columns show the pyruvate decarboxylase activities extracted from 24-hour growth cells.

TABLE 1

Amino Acid Analysis of Casamino Acids

\begin{tabular}{ccc}
\hline \hline $\begin{array}{c}\text { Lot of } \\
\text { Casamino acid }\end{array}$ & NR 1219 & $\mathrm{R} 69$ \\
\hline & \multicolumn{2}{c}{$\mu$ moles $/$ mg } \\
Asp & 0.4485 & 0.2286 \\
Thr & 0.2229 & 0.1847 \\
Ser & 0.3406 & 0.2885 \\
Glu & 0.5627 & 0.7368 \\
Pro & 0.6288 & 0.4954 \\
Gly & 0.5198 & 0.1268 \\
Ala & 0.5064 & 0.1797 \\
Val & 0.2861 & 0.2402 \\
Met & 0.0476 & 0.0788 \\
Ile & 0.1840 & 0.1510 \\
Leu & 0.3900 & 0.3904 \\
Tyr & 0.0110 & Trace \\
Phe & 0.0165 & 0.0104 \\
His & 0.1416 & 0.1113 \\
Lys & 0.3898 & 0.3932 \\
Arg & 0.1616 & 0.1251 \\
\hline
\end{tabular}

of protein per hr) with medium 1,2 , 3 and 4 respectively. The activity of the normal cells was about twice that with NR 1219, and the increase of PDC activity was also proportional to the amount of inositol in the medium (except the case of medium 1) as seen with NR 1219. The significantly high activity in the cells grown in the depleted medium with $\mathrm{R} 69$ is not understandable. It might explain the higher acetoin accumulation in the depleted medium with $\mathrm{R} 69$ than that with NR 1219.

3. Amino Acid Analyses of the Casamino Acids

The different responses in acetoin accumulation and PDC activity of the yeast to inositol by the lots of Casamino acids used as nitrogen sources in the medium might result from the different composition of amino acids in the hydrolysates. The results of amino acid analyses of the two lots of Casamino acids are given in Table 1. The contents of glycine, alanine and methionine differed considerablly between the two lots.

4. The Effect of Inositol when the Reconstituted Amino Acid Mixtures were Used as Nitrogen Sources

Two pure amino acid mixtures which are equivalent to Casamino acids, NR 1219 and $\mathrm{R} 69$, were reconstituted to replace the latters as nitrogen sources in the medium.

The effects of inositol on acetoin accumulation (Fig. 3) and PDC activity (Fig. 4) were compared between Casamino acid and the reconstituted amino acid mixtures. The broken lines in Fig. 3 represent acetoin levels in 24-hour culture medium with reconstituted amino acid mixtures and the dashed lines those with original Casamino acids. As clearly shown in the figure, the patterns of acetoin accumulation with reconstituted amino acid mixtures were in good coincidence with those in the original medium. Namely, R 69 groups 


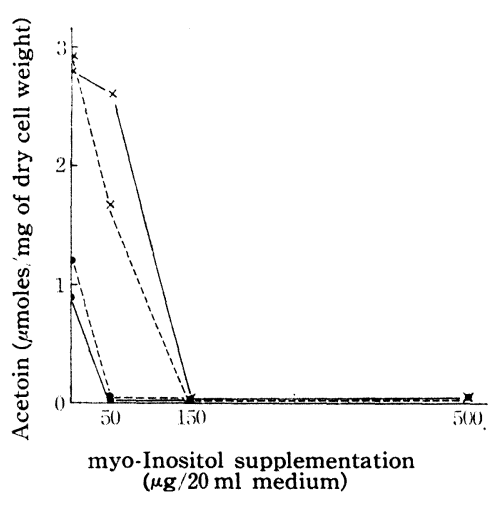

FIG. 3 The Comparison of Acetoin Levels in the Original Culture Medim with Those in the Reconstituted Ones

Saccharomyces carlsbergensis was cultivated at $30^{\circ}$ with shaking for 24 hours in the original media or the reconstituted ones.

The dashed lines indicate the acetoin levels in the original culture media and the broken lines indicate those in the reconstituted culture media.

- $=$, Casamino acid NR 1219; $\times \cdots \times$, Casamino acid R 69

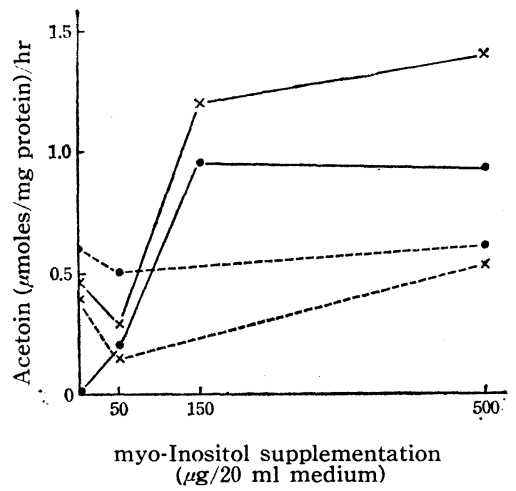

FIG. 4 The Comparison of Pyruvate Cecarboxylase Activity of the Cells Grown in the Original Media with Those Grown in the Reconstituted Ones

The dashed lines show the changes of pyruvate decarboxylase activity extracted from the 24hour growth cells in the original media (Casamino acid) and the broken lines those extracted from the 24-hour growth cells in the reconstituted media (equivalent amino acid mixtures).

-..., NR1219; $\times \ldots \times, \mathrm{R} 69$.

gave higher acetoin concentration in the culture medium than NR 1219 groups at low inositol concentration.

The patterns of PDC activity of the growth on the reconstituted medium, however, greatly differed from those in the original medium as shown in Fig. 4. The proportional changes in PDC activity to the increasing amount of inositol in the medium which were seen with the original casamino acid, could not be reproduced with the reconstituted amino acid mixtures as shown in broken lines. The change of the activity was observed only in small magnitude (0.52-0.64 (NR 1219 reconstituted), $0.15-0.55 \mu$ mole acetoin $/ \mathrm{mg}$ protein per hour ( $\mathrm{R} 69$ reconstituted) as the inositol level in the medium increased from 0 to $500 \mu \mathrm{g}$ per $20 \mathrm{ml}$ of the medium.

\section{Discussion}

As shown in Fig. 1, the growth of S. carlsbergensis 4228 became maximum and the amounts of acetoin produced in the culture medium was negligible when 150 $\mu \mathrm{g}$ of inositol per $20 \mathrm{ml}$ of the medium was present. There is no significant difference in the growth response by the lots of Casamino acids used (NR 1219 or $R$ 69) so this amount of inositol seems to be optimal for the normal growth of the yeast. The amounts of accumulated acetoin, however, was influenced by the Casamino acid reaching $2.80 \mu \mathrm{moles} / \mathrm{mg}$ of dry cell weight $(0.028$ in the normal culture) with $\mathrm{R} 69$, and $0.885 \mu \mathrm{mole} / \mathrm{mg}$ of dry cell weight ( 0.030 in the normal) with NR 1219 in 48 hour cultivation without inositol. The ratios of acetoin released 
by the deficient to the normal yeast with R 69 and NR 1219 were 100.0 and 29.5, respectively.

The specific activity of PDC and its response curve to the amounts of inositol was also different each other and the activity was generally higher with $\mathrm{R} 69$. An interesting observation is that the proportional increase of PDC activity to the amount of inositol observed in the yeast cultivation with R 69 or NR 1219 could not be reproduced when they were replaced by the equivalent amino acid mixture.

Witt et al. (1) have shown that de novo synthesis of PDC was induced by thiamine, and more effectively by thiamine together with $\mathrm{NH}_{4} \mathrm{Cl}$ in $S$. cerevisiae. The inductive rise of PDC activity by inositol, however, is different from "coenzyme induction", as inositol is not a coenzyme for PDC. The effect of $\mathrm{NH}_{4}{ }^{+}$ present in Casamino acid cannot be overestimated, as the basal medium contains $0.37 \%$ of $\left(\mathrm{NH}_{4}\right)_{2} \mathrm{SO}_{4}$. The study on an unknown factor in Casamino acid playing a supplementary role for PDC synthesis (or its activation) is now in progress.

\section{ACKNOWLEDGEMENT}

The authors express their deep gratitude to Dr. C. Kawasaki, Osaka University and Dr. E. Hayashi of this college for their continuous encouragement and helpful advices.

\section{REFERENCES}

1. Witt I., and Heilmeyer L., Biochim Biophys. Res. Comm., 25, 340 (1966).

2. Tomita T., Ozawa T., and Tomita I., J. Biochem., 65, 829 (1969).

3. Tomita T., Ozawa T., and Tomita I., Chem. Pharm. Bull., in press (1969).

4. Aoshima Y., Seikagaku, 29, 861 (1958).

5. Green D. E., Herbert D., and Subrahmanyan V., J. Biol. Chem., 138, 327 (1941).

6. Singer T.P., and Pensky J., Biochim. Biophys. Act., 9, 316 (1952).

7. Westerfeld W. W., J. Biol. Chem., 161, 495 (1945). 